\title{
Combined physical and statistical modeling of laser induced ultrasound signals from thin light absorbing films
}

\author{
Erika Svanström*, Tomas Linder*, and Johan E. Carlson ${ }^{\dagger}$ \\ ${ }^{*}$ EISLAB \\ Luleå University of Technology, SE-971 87 Luleå, Sweden \\ ${ }^{\dagger}$ Div. of Systems and Interaction \\ Luleå University of Technology, SE-971 87 Luleå, Sweden \\ Email: Johan.Carlson@ltu.se
}

\begin{abstract}
This paper presents a method for predicting the ultrasound pulses generated by thin semi-transparent polymer films, excited by a short laser pulse. The acoustic pressure is first modeled based on the physical properties of the polymer. Partial Least-Squares Regression is then used to link the model pressure to the ultrasound pulses measured by an ultrasound transducer. The uncertainty of the regression is also simulated, showing that the method is robust to noise in the measurements.
\end{abstract}

\section{INTRODUCTION}

Ultrasound is applied in various measurement techniques, e.g. in biomedical diagnostics, for materials characterization and non-destructive testing. Inducing ultrasound by a thermoelastic process is the most common mechanism for optical generation of ultrasound [1]. The mechanism rests on absorption of an incident laser light pulse and an energy conversion from electromagnetic waves to mechanical waves as ultrasound [2]. The conversion is through the thermoelastic process of heating leading to a corresponding pressure wave. This laser-induced ultrasound can be made broadband and of high frequency [3]. However, the conversion efficiency has been low, with low transmitted power [4]. Modeling the generated ultrasound could make up a basis for shaping the ultrasound according to specific measurement applications. Laser-induced ultrasound depend both on the input parameters as the light source, and on parameters of the material in which the light-to-sound conversion and sound propagation takes place, as described by Zhao et al. [5]. The material parameters are, for example, the optical absorption coefficient, the thermal expansion coefficient, the material density and the speed of sound. The laser, or input, parameters are the wavelength and the energy distribution in space and time. In the present work the laser-pulse is assumed short and modeled as a delta pulse, and the physical properties of the materials play a more prominent role in the modeling.

A thermoelastic model of laser-generated acoustic pressure waves from thin layers of optically absorbing Polyethylene Terephthalate (PET) is compared with measurements to determine the voltage responses from a polymer (PVDF) transducer. A physical model is first developed to predict the acoustic pressure wave generated in the thin film. The second step is then to link the transducer output voltage to the pressure wave, i.e. to account for the combined effects of the propagation medium and the transducer's electro-mechanical transfer function. The results are then compared with experimentally measured output voltages, $v(t)$.

The overall objective is to be able to predict the output voltage resulting from exciting a thin PET layer with known material characteristics, with a short laser pulse, thus facilitating the customization and optimization of such optoacoustic setups.

The properties of the thin film were varied in a designed experiment consisting of 9 different configurations and replicates, resulting in a total of 14 experiments. For each of the configurations, the theoretical sound pressure, $p(t)$, was determined by a solution of the 1D photoacoustic wave equation for thermoelastic expansion in a light-absorbing layer of a laser pulse. The link between the modeled pressure and the measured transducer output voltage $v(t)$, was then established through a multivariate calibration technique called Partial Least-Squares Regression (PLSR) [6], [7].

The paper presents the results of the measured and the modeled voltages for the PET film configurations given the acoustic pressure, $p(t)$, determined from the material properties of the thin PET film. Along with these results, analysis of the uncertainty and predictive performance of the multivariate calibration scheme are presented.

\section{THEORY}

\section{A. Photoacoustic wave equation}

To model the sound pressure $p(x, t)$ at position $x$ at time $t$, the one-dimensional photoacoustic wave equations [8]

$$
\frac{\partial^{2} p_{i}(x, t)}{\partial x^{2}}-\frac{1}{c_{i}^{2}} \frac{\partial^{2} p_{i}(x, t)}{\partial t^{2}}=-\frac{\beta_{i}}{C_{p i}} \frac{\partial H_{i}(x, t)}{\partial t}
$$

were solved in layers $i=1,2,3$, with Heating functions

$$
H_{1} \equiv 0, H_{2}(x, t)=\mu_{a 2} \mathrm{e}^{-\mu_{a 2} x} E_{02} \delta(t), H_{3} \equiv 0
$$

of a light-absorbing layer $i=2$ surrounded by two transparent materials. Assumptions were a short heating giving thermal and pressure confinement [8]. Boundary conditions were according to acoustic conservation laws for pressure and acceleration at interfaces [9]

$$
p_{i}\left(b_{i, i+1}\right)=p_{i-1}\left(b_{i, i+1}\right),
$$




$$
-\frac{1}{\rho_{i}} \frac{d}{d x} p_{i}\left(b_{i, i+1}\right)=-\frac{1}{\rho_{i-1}} \frac{d}{d x} p_{i+1}\left(b_{i, i+1}\right)
$$

and by choice $0 \leq b_{1,2}$, and $b_{1,2}<b_{2,3}$. Initial conditions were

$$
\begin{aligned}
p_{i}\left(x, 0_{-}\right) & =0 \\
\frac{\partial}{\partial t} p_{i}\left(x, 0_{-}\right) & =0 \\
H_{i}\left(x, 0_{-}\right) & =0 .
\end{aligned}
$$

The solution approach to Eq. (1) was by using the Laplace transform as an expansion of calculations by Shan et al. [10] into applying two boundary interfaces. The solution also included a geometric series expansion in handling periodic terms.

\section{B. Analytical sound pressure expressions}

The acoustic pressure $p_{3}(x, t)$ in layer 3 is expressed as

$$
\begin{gathered}
p_{3}(x, t)=\sum_{j=1}^{N} p_{3_{j}}(x, t), \\
p_{3_{j}}(x, t)=\frac{F_{2}\left(R_{23}+1\right)}{2} \cdot \theta\left(x-b_{2,3}\right) \\
\cdot\left(p_{3 j \text { even }}(x, t)+p_{3 j \text { odd }}(x, t)\right)
\end{gathered}
$$

with collecting constant

$$
F_{2}=\frac{\mu_{a 2} \beta_{2} E_{02} c_{2}^{2}}{C_{p 2}}
$$

and with the Heaviside $\theta\left(x-b_{2,3}\right)$ specifying the $x$ domain for layer $i=3$. The reflection coefficients at interfaces towards layer 1 and 3 of waves in layer $i=2$ reflected back towards layer 2 , were

$$
R_{21}=\frac{c_{1} \rho_{1}-c_{2} \rho_{2}}{c_{2} \rho_{2}+c_{1} \rho_{1}}, R_{23}=\frac{c_{3} \rho_{3}-c_{2} \rho_{2}}{c_{2} \rho_{2}+c_{3} \rho_{3}},
$$

respectively. Note that a change in polarity of the sound pressure is given by a reflection coefficient of negative sign, from a reflection against a material with a smaller impedance $Z_{i}=c_{i} \rho_{i}$.

Acoustic pressure wave components were

$$
\begin{array}{r}
p_{3 \text { j even }}(x, t)=\left(R_{21} R_{23}\right)^{j-1} \cdot \mathrm{e}^{\mu_{a 2} c_{2}\left(t_{j \text { even }}+t-\frac{b_{2,3}}{c_{2}}\right)} \\
\cdot \theta\left(t_{\text {jeven }}+t\right) \cdot \theta\left(-t_{\text {jeven }}\right) \cdot \theta\left(-t_{\text { even }}-t-\frac{b_{1,2}-b_{2,3}}{c_{2}}\right), \\
p_{3 j \text { odd }}(x, t)=\frac{\left(R_{21} R_{23}\right)^{j}}{R_{23}} \cdot \mathrm{e}^{c_{2} \mu_{a 2}\left(-t_{\text {jodd }}-t-\frac{b_{1,2}}{c_{2}}\right)} \\
\cdot \theta\left(t_{j \text { odd }}+t\right) \cdot \theta\left(-t_{j \text { odd }}\right) \cdot \theta\left(-t_{j \text { odd }}-t-\frac{b_{1,2}-b_{2,3}}{c_{2}}\right) \cdot
\end{array}
$$

Even and odd number of reflections within layer 2 are expressed by

$$
\begin{gathered}
t_{j \text { even }}=t_{\text {arg }}(2 \cdot(j-1)), \\
t_{j \text { odd }}=t_{\text {arg }}(2 \cdot j-1),
\end{gathered}
$$

employing even and odd arguments $j_{\text {arg }}$ in

$$
t_{\text {arg }}\left(j_{\text {arg }}\right)=\frac{b_{2,3}-x}{c_{3}}+\frac{j_{\text {arg }} \cdot\left(b_{1,2}-b_{2,3}\right)}{c_{2}} .
$$

The Heaviside functions $\theta\left(t_{\text {arg }}+t\right)$ and $\theta\left(-t_{\text {arg }}\right)$ originate in the Laplace inverse transformation, and

$$
\theta\left(-t_{\text {arg }}-t-\frac{b_{1,2}-b_{2,3}}{c_{2}}\right)
$$

limits the function domain of the $j$ component $p_{3_{j}}(x, t)$ towards the $(j+1)$ wave component in the sum in Eq. (8).

\section{Multivariate calibration scheme}

To connect the modeled acoustic pressures to the measured voltages, we apply a multivariate calibration technique (i.e. regression method) called Partial Least-Squares Regression (PLSR). The PLS method was developed by Herman Wold [6], and has been applied in many areas of experimental science. The details of the PLS calculations can be found in [11] and [7]. Here, only a brief summary is given.

Consider the general linear model,

$$
\mathbf{Y}=\mathbf{X A} \text {, }
$$

where the $\mathrm{Y}$ bock is a matrix containing the properties we would like to estimate (in this case the measured voltages), given a set of descriptors (X block, i.e. the modeled sound pressures) and some regressor matrix, A. The task of any regression method is thus to find the matrix $\mathbf{A}$ that yield the best estimate of $\mathbf{Y}$, in some sense, i.e.

$$
\widehat{\mathbf{Y}}=\mathbf{X} \widehat{\mathbf{A}},
$$

where $\widehat{\cdot}$ denotes an estimated quantity.

The central idea of PLS is to determine a common set of basis functions (PLS components) for both the $\mathrm{X}$ block and the $\mathrm{Y}$ block, so that they best describe the cross-covariance between the blocks. In this work, the $\mathrm{X}$ block is a matrix which columns are the measured quantities, i.e. the sampled voltage signals at the ultrasound transducer. The Y block is a matrix which columns are the modeled acoustic pressures from Eq. (8)

1) Estimating $\mathbf{Y}$ from $\mathbf{X}$ : Using Eqs. (3)-(11) in [11], the $\mathrm{X}$ block and $\mathrm{Y}$ block are first transformed into their PLS component representation,

$$
\begin{aligned}
& \mathbf{X}=\mathbf{T} \mathbf{P}^{T}+\mathbf{E} \\
& \mathbf{Y}=\mathbf{T Q}^{T}+\mathbf{F}
\end{aligned}
$$

where $\mathbf{E}$ and $\mathbf{F}$ are residual matrices of the $\mathrm{X}$ and $\mathrm{Y}$ blocks, respectively. Given an existing set of PLS components, the estimate of $\mathbf{Y}$ is given by

$$
\widehat{\mathbf{Y}}=\mathbf{X} \widehat{\mathbf{A}},
$$

where $\widehat{\mathbf{A}}=\tilde{\mathbf{W}} \mathbf{Q}^{T}$ and $\tilde{\mathbf{W}}$ and $\mathbf{Q}$ are given by Eqs. (15) and (11) in [11], respectively. Here, the matrix $\tilde{\mathbf{W}} \mathbf{Q}^{T}$ is determined from the calibration experiments in Table I, and later used to estimate $\widehat{\mathbf{Y}}$. 


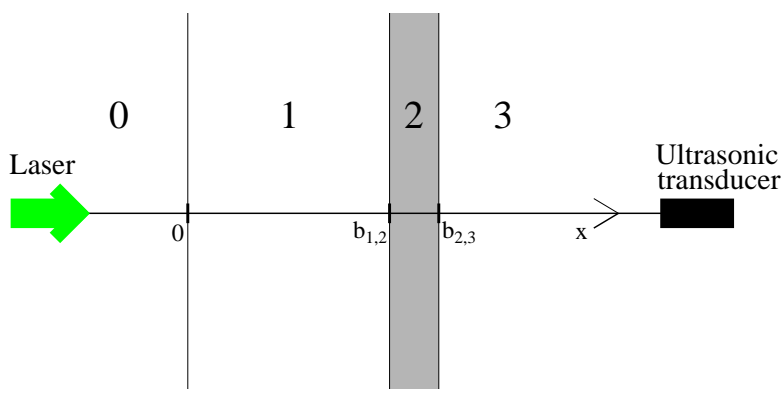

Fig. 1. Setup of experiment and of theoretical model. Material layer 0 as well as 3 are water, 1 is glass, and 2 is light-absorbing PET polymer film. Material layers 1,2,3 are modeled theoretically.

2) Determining PLS model order: As in all modeling, finding an appropriate model order is an important, and difficult, problem. There are numerous criteria available for model order selection, some which tend to overestimate the model-order, while others are overly conservative [12]. For PLS modeling problems, cross-validation is the most commonly used method [13], which is what we used here. The model order was evaluated by looking at the $Q^{2}$ statistic [14] as a function of the number of components in the PLS model, withholding one experiment at a time. For our experiments, we found that five PLS components is suitable.

\section{EXPERIMENTS}

Experiments were performed on laser-induced ultrasound in samples of a layer of stacked dyed PET polymer film mounted on glass substrate and immersed in water [15], see Fig. 1. The ultrasound was recorded by a broadband PVDF transducer. The physical properties of absorption coefficient and absorber layer thickness were varied. The thickness was varied by changing the number of layers of a $50 \mu \mathrm{m}$ film.

In the experiment, pulsed laser light of wavelength $\lambda=532$ $\mathrm{nm}$ and pulse length $9 \mathrm{~ns}$ was sent into a basin of water. The laser light source was a system NL 202/SH from EKSPLA, Lithuania, as a diode pumped, Q-switched, frequency doubled, $\mathrm{Nd}$ :YAG laser. In the basin a sample of a semi-transparent absorbing layer mounted on a glass substrate was placed perpendicularly to the beam axis. As light-absorbing layer a planar, dyed, non-conductive, semi-transparent, Polyethylene Terephthalate (PET) polymer film in a stack of one up to four films was used. The film thicknesses were all $50 \mu \mathrm{m}$, and the optical absorption coefficients $\mu_{a}=11 \cdot 10^{3}, 18 \cdot 10^{3}$, $27 \cdot 10^{3}$ and $44 \cdot 10^{3} \mathrm{~m}^{-1}$ respectively. The laser pulse energy absorbed by the layer was converted to an ultrasonic pulse through a thermoelastic process in the layer. The ultrasonic pulse propagated through the water and was recorded by an immersed broadband ultrasonic PVDF (polyvinylidene difluoride) transducer. For each experimental run, data from 10 individual laser pulses were recorded for subsequent signal processing. The recorded voltage time signal was processed in MATLAB, The Mathworks Inc., MA, USA. To reduce the influence from noise, the ultrasonic pulses of interest are windowed and according to Grennberg et al. [16] aligned, to reduce the timing jitter, and then averaged. The averaged time signal were thereafter Fourier transformed to give frequency spectra.
The experimental design is given in Table I.

TABLE I. EXPERIMENTAL DESIGN. THE BOXES INDICATE THE EXPERIMENTS STUDIED IN MORE DETAIL IN THE SEC. IV

\begin{tabular}{|ccc|} 
Exp. no & No. of layers & $\mu_{a}\left[\mathrm{~m}^{-1}\right]$ \\
\hline \hline 1 & 3 & $44 \times 10^{3}$ \\
2 & 2 & $18 \times 10^{3}$ \\
\hline 3 & 1 & $11 \times 10^{3}$ \\
\hline 4 & 3 & $11 \times 10^{3}$ \\
\hline 5 & 2 & $18 \times 10^{3}$ \\
\hline 6 & 1 & $11 \times 10^{3}$ \\
\hline 7 & 3 & $44 \times 10^{3}$ \\
\hline 8 & 1 & $44 \times 10^{3}$ \\
\hline 9 & 1 & $11 \times 10^{3}$ \\
\hline 10 & 3 & $11 \times 10^{3}$ \\
\hline 11 & 1 & $18 \times 10^{3}$ \\
12 & 1 & $27 \times 10^{3}$ \\
13 & 2 & $11 \times 10^{3}$ \\
14 & 4 & $11 \times 10^{3}$ \\
\hline
\end{tabular}

\section{RESUlTS}

Figures 2, 3, 4, and 5 show the results of using PLS regression to connect the modeled acoustic pressure to the measured voltages (at the PVDF receiver), for experiments no. 3, 5, 8, and 10 (see Table I), respectively.

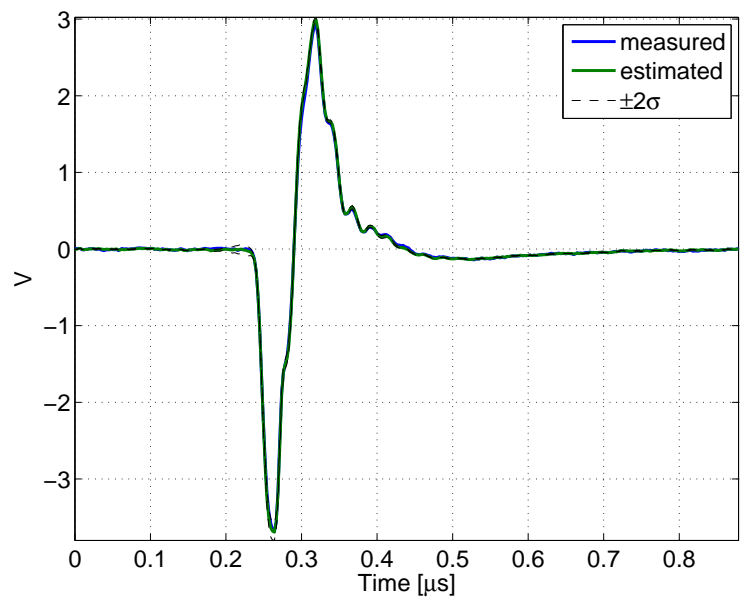

Fig. 2. Experiment no. 3 in Table I

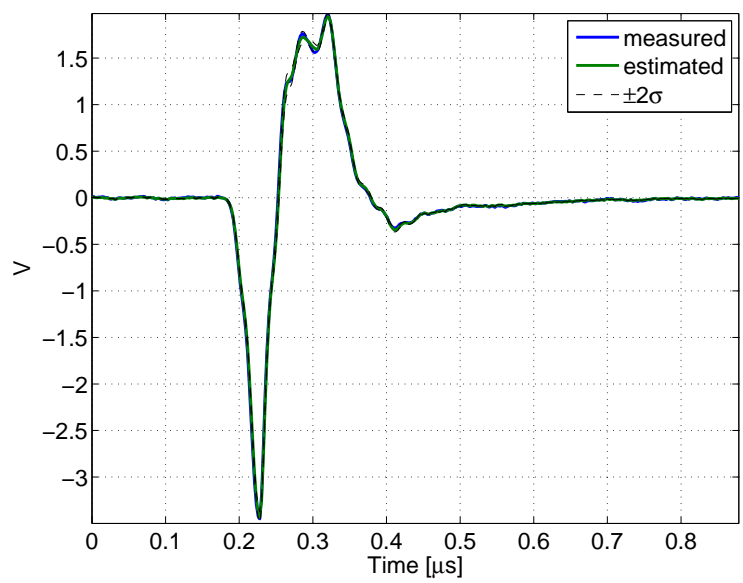

Fig. 3. Experiment no. 5 in Table I 


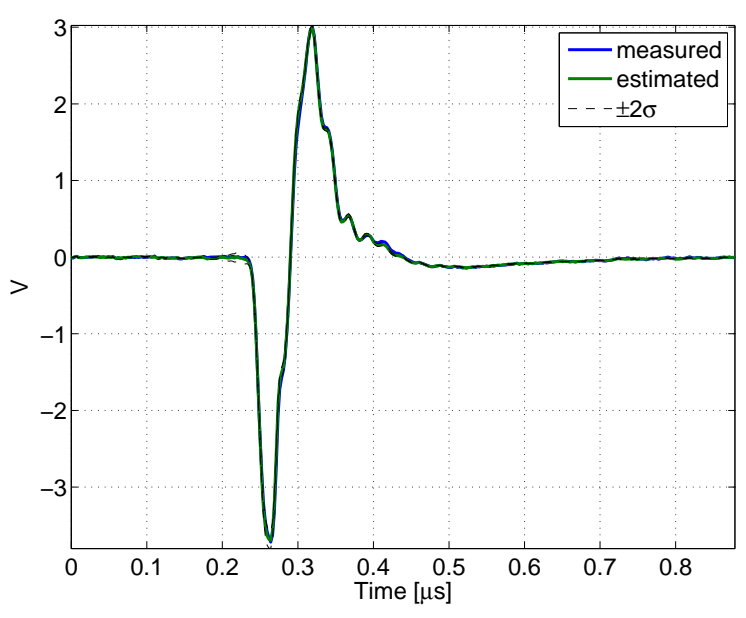

Fig. 4. Experiment no. 8 in Table I

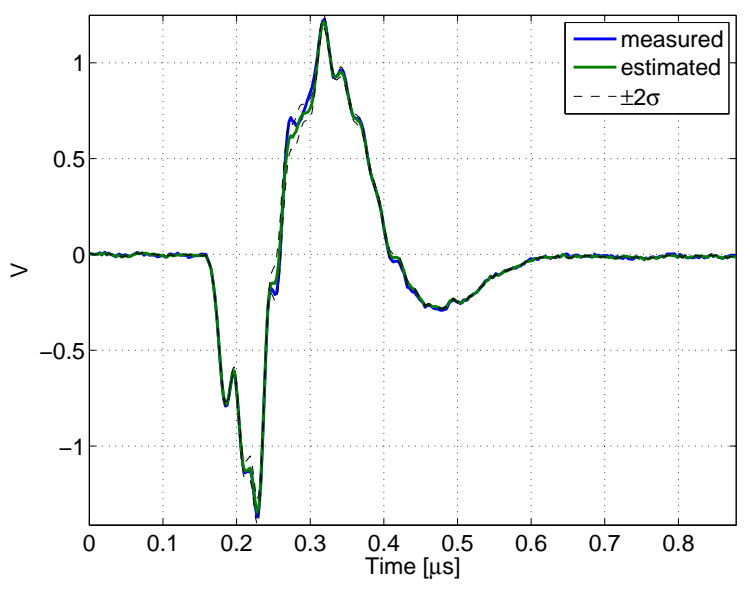

Fig. 5. Experiment no. 10 in Table I

In each of the figures, the measured voltages and the voltages estimated using PLSR are plotted. Also, although difficult to see, a $\pm 2 \sigma$ interval is plotted for the estimated voltages. This interval was simulated based on the assumption of an uncertainty in the voltage measurement of 5\% (of the maximum measured voltage). The details of the simulation are described in [17]. This is to test how sensitive the multivariate calibration is to experimental noise, and it is clear from these simulation that the estimation is very robust.

\section{CONCLUSION}

In this paper we have presented a method for modeling the ultrasound pulses induced by exciting a thin semi-transparent film with a short laser pulse. The model is based on a combination of a solution of the 1D photoacoustic wave equation and PLS regression. The physical model predicts the acoustic pressures generated in the film and the statistical regression model captures the electro-mechanical transfer function and the transfer function of the medium, thus linking the modeled sound pressures to the observed voltage signals.

The results show that the method works well for modeling a range of measurement configurations, which enables the user to predict the performance of a new setup, hence facilitating optimization of the configuration.

The uncertainty of the regression modeling was simulated using a Monte Carlo approach, and was found to be very low, indicating that the proposed method is robust to uncertainties in the measurement.

\section{REFERENCES}

[1] Y. Hou, S. Ashkenazi, S.-W. Huang, and M. O'Donnell, "Improvements in optical generation of high-frequency ultrasound," IEEE Trans. on Ultras., Ferroelec. and Freq. Contr., vol. 54, no. 3, pp. 682-686, 2007.

[2] V. E. Gusev and A. A. Karabutov, Laser optoacoustics. New York: AIP Press, 1993.

[3] M. O'Donnell, Y. Hou, J.-S. Kim, S. Ashkenazi, S.-W. Huang, and L. J. Guo, "Optoacoustic generation of high frequency sound for 3d ultrasonic imaging in medicine," The European Physical Journal Special Topics, vol. 153, no. 1, pp. 53-58, 2008.

[4] S. Ashkenazi, Y. Hou, S.-W. Huang, T. Buma, and M. O'Donnell, Photoacoustic Imaging and Spectroscopy, ch. High frequency optoacoustic transducers for ultrasonic and photoacoustic imaging, pp. 223238. CRC Press, 1 ed., 2009.

[5] Z. Zhao, S. Nissila, O. Ahola, and R. Myllylä, "Production and detection theory of pulsed photoacoustic wave with maximum amplitude and minimum distortion in absorbing liquid," IEEE Trans. on Instr. and Meas., vol. 47, no. 2, pp. 578-583, 1998.

[6] H. Wold, Systems Under Indirect Observation, Vols I, II. Amsterdam: North Holland, 1982.

[7] S. Wold, M. Sjöström, and L. Eriksson, "PLS regression: A basic tool of chemometrics," Chemometrics Intell. Lab. Syst., vol. 58, pp. 109-130, 2001.

[8] V. Lihong and W. Hsin-i, Biomedical optics : principles and imaging. Hoboken, N.J.: Wiley-Interscience, 2007.

[9] G. J. Diebold, M. I. Khan, and S. M. Park, "Photoacoustic "Signatures" of particulate matter: Optical production of acoustic monopole radiation," Science, vol. 250, no. 4977, pp. 101-104, 1990.

[10] Q. Shan, A. Kuhn, P. A. Payne, and R. J. Dewhurst, "Characterisation of laser-ultrasound signals from an optical absorption layer within a transparent fluid," Ultrasonics, vol. 34, no. 6, pp. 629-639, 1996.

[11] J. E. Carlson and R. Carlson, "Prediction of molar fractions in twocomponent gas mixtures using pulse-echo ultrasound and PLS regression," IEEE Trans. Ultrason., Ferroelec., and Freq. Contr., vol. 53, no. 3, pp. 606-613, 2006.

[12] J. Shao, "Linear model order selection by cross-validation," J. Am. Stat. Assoc., vol. 88, pp. 486-494, June 1993.

[13] I. N. Wakeling and J. J. Morris, "A test of significance for partial least squares regression," J. Chemom., vol. 7, no. 4, pp. 291-304, 1993.

[14] R. Carlson and J. E. Carlson, Design and Optimization in Organic Synthesis: Second Enlarged and Revised Edition. Amsterdam: Elsevier, 2nd ed., 2005.

[15] E. Svanström, T. Löfqvist, and J. Delsing, "On laser-induced ultrasound generated in a thin semi-transparent layered polymer structure," in Proc. of SPIE, the International Society for Optical Engineering, (San Fransisco, CA, USA), Feb 2012.

[16] A. Grennberg and M. Sandell, "Estimation of Subsample Time Delay Differences in Narrowband Ultrasonic Echoes Using the Hilbert Transform Correlation," IEEE Trans. Ultrason., Ferroelec., and Freq. Contr., vol. 41, no. 5, pp. 588-595, 1994.

[17] J. E. Carlson, J. Martinsson, and M. Lundberg Nordenvaad, "Measurement of methane content in upgraded biogas using pulse-echo ultrasound," in Proc. of International Congress on Ultrasonics, (Vienna, Austria), p. Paper ID 1366, April 9-12 2007. 